\title{
A Mixed-Flow Cellular Automaton Model for Vehicle Nonstrict Priority Give-Way Behavior at Crosswalks
}

\author{
Yunxuan Li $\mathbb{D}^{1,2,3}$ Zeyang Cheng $\mathbb{D}^{1,2,3}$ Jian Lu $\mathbb{D}^{1,2,3}$ and Lin Zhang ${ }^{4}{ }^{4}$ \\ ${ }^{1}$ School of Transportation, Southeast University, Nanjing, Jiangsu 211189, China \\ ${ }^{2}$ Jiangsu Key Laboratory of Urban ITS, Southeast University, Nanjing, Jiangsu 211189, China \\ ${ }^{3}$ Jiangsu Province Collaborative Innovation Center of Modern Urban Traffic Technologies, Southeast University, Nanjing, \\ Jiangsu 211189, China \\ ${ }^{4}$ School of Civil Engineering and Transportation, South China University of Technology, Guangzhou 510641, China \\ Correspondence should be addressed to Jian Lu; lujian_1972@seu.edu.cn
}

Received 9 November 2019; Revised 23 November 2020; Accepted 10 December 2020; Published 29 December 2020

Academic Editor: Meaad Saberi

Copyright (c) 2020 Yunxuan Li et al. This is an open access article distributed under the Creative Commons Attribution License, which permits unrestricted use, distribution, and reproduction in any medium, provided the original work is properly cited.

\begin{abstract}
The vehicle nonstrict priority give-way behavior (VNPGWB) is a common part of traffic interaction between motorized and nonmotorized vehicles in many countries. This study proposes a mixed-flow cellular automaton model to simulate the passing of vehicles in front of bicycles at crosswalks. The mixed-flow model combines a vehicle model with a bicycle model, using nonstrict priority give-way and strict give-way two driving behaviors defined as relating to the decision point rule and the launching rule, respectively. Simulation results showed that as the vehicle and bicycle inflow rates increased, a critical inflow rate divided vehicle and bicycle traffic flow into free flow and saturated flow conditions. The values of vehicle saturation flow decreased from 0.34 to 0.05 , and the values of bicycle saturation flow decreased from 0.54 to 0.44 , indicating that the mixed traffic flow has a negative effect on vehicle and bicycle saturated flow. Results also showed that VNPGWB effectively improves vehicle saturation flow over that of the strict give way. The advantage of VNPGWB is more significant when vehicles and bicycles are in saturation traffic flow.
\end{abstract}

\section{Introduction}

In many countries in Europe and Asia, the bicycle is still an important mode of transportation. For example, in China, more than $38 \%$ of commuters choose bicycles as their main travel mode $[1,2]$. It is noteworthy that electric bicycles have grown rapidly in recent years, with the number of e-bikes exceeding 250 million in China [3, 4]. In addition, shared bicycles also have gained tremendous popularity as a result of their convenience $[5,6]$. However, due to their vulnerability in a collision, cyclists are subject to higher safety risks than drivers of vehicles $[7,8]$. Collisions between bicycles (including e-bikes) and vehicles tend to cause very severe injuries and fatalities. Some studies show that $90 \%$ of all cyclist fatalities are caused by collision with vehicles [6, 9-11]. In fact, vehicles and bicycles competing for priority are a common reason for conflicts and crashes at crosswalks [12]. Most traffic managers believe that drivers should strictly give way to bicycles passing through a crosswalk $[3,13,14]$. However, actual investigations have found that drivers in many countries and regions, such as Norway, Finland, Germany, and China, do not strictly adhere to this rule $[8,15-19]$. That is, during the real traffic conditions, vehicle drivers may not always assume they must comply with the rule of giving way, and instead, they compete for priority $[17,18]$. For example, the decision a driver makes to pass through the crosswalk or not depends on the bicycle's position and speed. Since it is based on the driver's subject assessment, the behavior is defined as vehicle nonstrict priority give-way behavior (VNPGWB).

In the process of vehicles passing in front of bicycles at crosswalks, drivers have only two choices: passing without stopping or stopping to give way [17]. Drivers should generally adapt their speeds to avoid endangering cyclists at crosswalks, and if necessary, drivers should stop to give way to cyclists [12]. This rule ensures the orderly passing of 
vehicles and bicycles at the crosswalk. However, when the vehicle and bicycle are in a condition of saturated traffic flow, the vehicle flow is usually blocked by the bicycle flow, which has a significant impact on the traffic capacity of the vehicle flow, especially near unsignalized intersections $[15,17]$. Specifically, there is a great impact on traffic flow when vehicles are waiting for bicycles at crosswalks. In this case, nonstrict priority allows vehicles to leave the conflict area earlier, mitigating their effects on subsequent vehicles and thereby improving the capacity of the road and intersection. Therefore, despite the negative impact on traffic safety, many countries acquiesce to nonstrict priority in the practical management of traffic, administering no punishment for violators as long as no crash occurs [17, 19].

This study uses a cellular automaton (CA) model to simulate a vehicle passing in front of bicycles at crosswalks engaged in VNPGWB. In order to understand VNPGWB, the key focus is to combine a mixed-flow model (i.e., integrating a vehicle model and a bicycle model) with driving behaviors. Driving behaviors in this study are primarily divided into the decision point rule and the launching rule. By adjusting the proportion of driving behaviors (strict give way and nonstrict priority give way) in these two rules, the real traffic conditions for VNPGWB can be simulated.

The rest of this paper is organized as follows: Section 2 reviews the literature; Section 3 describes the proposed mixed method in detail; Section 4 measures numerical simulation; and Section 5 concludes the paper.

\section{Literature Review}

Few previous studies explored VNPGWB, Räsänen [16] was the first to examine the behavior of road users changed based on their knowledge of priority regulations, such as whether a turning vehicle needs to give way to a cyclist from the same or opposite direction. Results showed that the effect of priority regulations on road user behavior depended on the characteristics of the bicycle crossings. Most subsequent studies found that drivers choose a give-way behavior depending on their understanding of priority order. For example, Lin et al. [17] developed a microdriving force model, which included safety driving force and efficiency driving force, for right-turning drivers, who constitute the dominant group confronted with the nonstrict priority passing situation. Silvano et al. [7] presented a modeling framework to describe driver-cyclist interactions when they were approaching a conflict zone. In the framework, driver yielding, or give-way, behavior is modeled as a function of several explanatory variables. Ma et al. [8] established a three-layered mathematical model, including a decision layer, operation layer, and constraint layer, to simulate the variation in trajectories of right-turn vehicles. Bai et al. [19] estimated the capacity of left-turn vehicles under nonstrict priority. Results showed that the model was valid for estimating the capacity of an exclusive left lane with a permitted phase under nonstrict priority. In summary, most studies have assumed that all drivers follow VNPGWB; however, drivers who choose to strictly give way coexist in mixed traffic flow with those who do not. Further, a given driver may make different decisions under free flow and saturated flow conditions.

When passing bicycles at crosswalks, most drivers constantly adjust their vehicles' speed. In order to simulate this behavior, this study will establish a microscopic simulation model. The most popular microscopic simulation methods used in previous studies are car-following models and cellular automaton (CA) models [20-22]. Because the car-following model primarily simulates the interaction between a front and rear vehicle, the model is only applicable to one-dimensional movement and cannot represent the full mixed traffic flow. The CA traffic flow model, in contrast, can make full use of computer operations to flexibly change its rules according to various traffic conditions. In fact, the movements of vehicles and bicycles are discrete, and the CA model can use discrete space-time and state variables to regulate evolution rules and thus to describe nonlinear behavior. Additionally, the CA model can simulate the gradual change in vehicle and bicycle behavior as conditions change from free flow to saturated flow [23-25]. It can be found, through long-term simulation, whether there is a phase transformation from free flow to saturation flow. In recent years, a large number of studies have used CA models to simulate mixed traffic flow. For example, Meng et al. [26] proposed a single-lane CA model to simulate mixed traffic with motorcycles and investigated the relationship between motorcycle lane changing behavior and density of traffic flow. Zhao [24] established a mixed bicycle traffic model, comprised of two bicycle types of bicycles, that shows bicycle traffic characteristics on eight physically separated bicycle paths in China. Ren et al. [21] improved a cellular automaton model by incorporating social forces that can describe interactions between pedestrians, making it useful for modeling the bidirectional pedestrian flow at crosswalks. Lu et al. [25] proposed a simulation model to represent vehicles yielding to pedestrians at crosswalks and demonstrated the relationship between saturated flow and yield behavior. The CA model can demonstrably be applied to simulate the behavior of vehicle drivers passing bicycles at crosswalks.

\section{Model}

The crosswalk is one of the most serious conflict areas between vehicles and bicycles. Obviously, cyclists are vulnerable road users in this conflict area. In order to avoid a crash, a vehicle driver's decision process begins from upstream of the conflict area, where the driver confirms whether there is a potential conflict or not. The decision process of giving way behavior can be divided into two situations, as shown in Figure 1. Situation 1 (Figures 1(a)1(c)): the white car (red box) has observed the bicycle (red box) at the crosswalk and chooses to pass without giving way at all; Situation 2 (Figures 1(d)-1(f)): the blue car (red box) has observed the bicycle (red box) at the crosswalk and stops to give way. Based on field observation, a vehicle's passing through bicycles at the crosswalk can be divided into the following steps: (1) the vehicle reaches the decision point; (2) the vehicle stops outside the conflict area; (3) the vehicle 


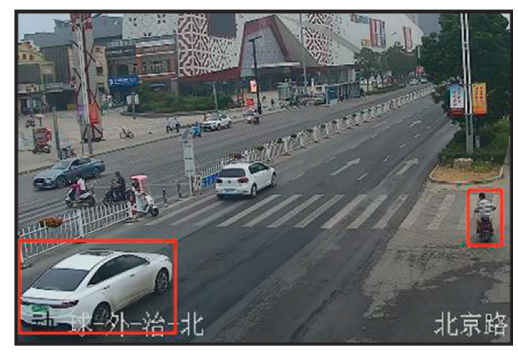

(a)

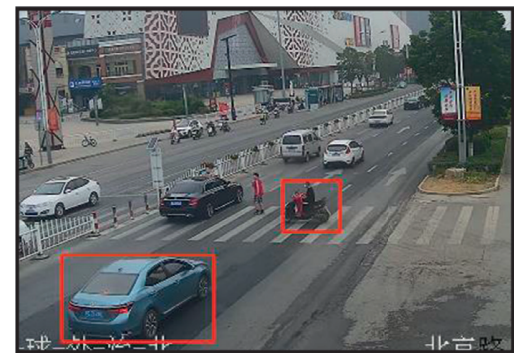

(d)

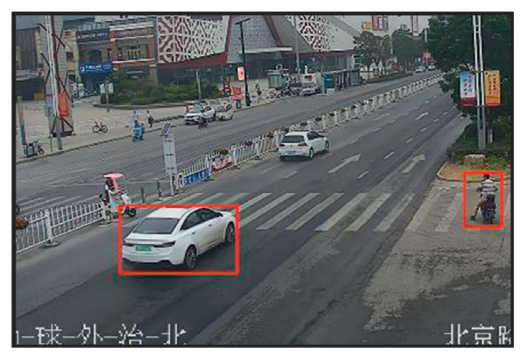

(b)

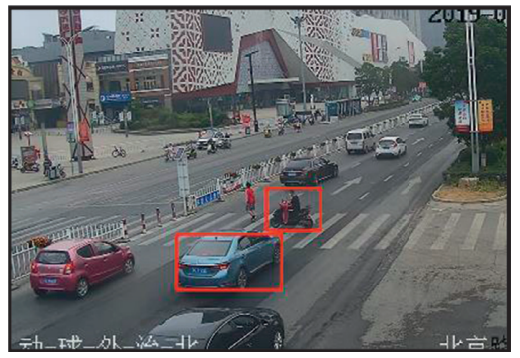

(e)

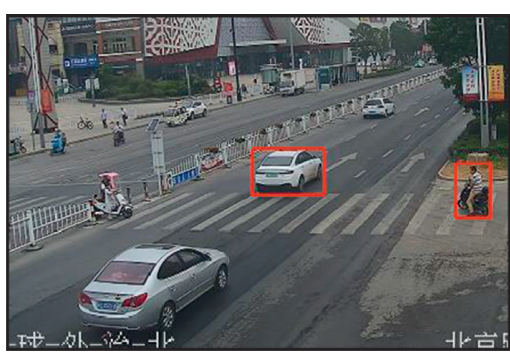

(c)

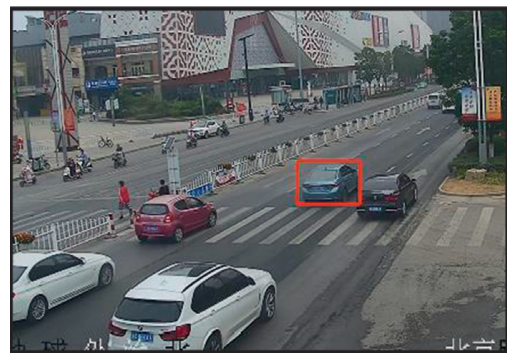

(f)

FIGURE 1: The process of vehicles passing bicycles at crosswalks in real traffic condition.

passes through the conflict area. It is worth noting that VNPGWB only occurs in Steps 1 and 2. The schematic diagram of these steps is depicted in (Figures 2(a)-2(c)). When a vehicle needs to pass through a crosswalk, the driver's decision process begins at a certain distance upstream of the conflict area, a distance defined as the decision point (Figure 2(a)). When a vehicle stops to give way to a bicycle, the driver usually adjusts speed and stops at the boundary of the conflict area. Once an acceptable gap exists, the driver will finish the traverse. The stop position in this study is defined as the launching point (Figure 2(b)). However, if the driver chooses to pass without giving way, Step 2 will not occur.

3.1. Definition and Delimitation of Cellular Space. A vehicle lane and a crosswalk were chosen as the research area for this study. First, the cellular space is defined as a two-dimensional matrix, and the $n$th cellular unit in the cellular space position is $\left(x_{n}, y_{n}\right)$. Then, because of the different sizes of vehicles and bicycles, a finer cellular unit size is defined in order to comply with the actual speed and minimum vehicle space for each. One bicycle occupies two cellular units $\left(x_{n}^{b}, y_{n}^{b}\right)$ and one vehicle $\left(x_{n}^{v}, y_{n}^{v}\right)$ occupies $6 \times 4$ cellular units. The vehicle lane widths are $l^{v} \mathrm{~m}$ and the bicycle lane widths are $l^{b} \mathrm{~m}$; thus, the overlap area between the vehicle lane and the bicycle lane is $l^{v} \times l^{b} \mathrm{~m}^{2}$. Finally, the conflict area in the cellular space position is shown in Figure 3.

3.2. Vehicle Model. According to the vehicle's position in its lane, the vehicle model can be divided into three rules: the driving rule, the decision point rule, and the launching rule. When following the driving rule, the driver selects an acceptable distance in understanding the interaction rules with consideration of safety and other factors. Then, the decision point rule is used: when drivers observe the bicycle lanes, different drivers (strict give way and nonstrict priority give way) make different decisions about whether to give way. If the vehicle slows down and stops at the boundary of the conflict area, it will be launched through the area when there is an acceptable gap. The launching rule is used to determine the acceptable gap for the different types of drivers making different decisions.

3.2.1. Vehicle Rule 1: Driving Rule. This study assumes that the vehicle is not affected by lane changes and reverse lanes. All vehicles move at an expected maximum velocity, and they adjust their velocity in order to avoid collisions with vehicles in front of them. For a process of $t \longrightarrow t+1$, the driving rule evolution is as follows:

$$
\begin{aligned}
& \text { Step 1: acceleration; } v_{n}^{v} \longrightarrow \min \left(v_{n}^{v}+a^{v}, v_{\max }^{v}\right) \\
& \text { Step 2: slowing down; } v_{n}^{v} \longrightarrow \min \left(v_{n}^{v}, d_{n}^{v}\right) \\
& \text { Step 3: randomization with probability } p_{s} \text {; if rand }>p_{s} \text {, } \\
& \text { then } v_{n}^{v} \longrightarrow \max \left(v_{n}^{v}-a^{v}, 0\right) \\
& \text { Step 4: movement; } x_{n}^{v} \longrightarrow x_{n}^{v}+v_{n}^{v},
\end{aligned}
$$

where $x_{n}^{v}$ and $v_{n}^{v}$ represent the position and velocity, respectively, of vehicle $n ; a^{v}$ represents vehicle acceleration; $d_{n}^{v}=x_{n+1}^{v}-x_{n}^{v}-l^{v}$ is the number of cellular spaces between vehicle $n$ and vehicle $n+1 ; l^{v}$ is the length of the vehicle; and randis a random number between 0 and 1 , where $p_{s}$ is the randomization with probability.

This model adopts an open boundary: when vehicles' road position is updated, the positions of the head vehicle and the tail vehicle are monitored as $x_{\text {lead }}^{v}$ and $x_{\text {last }}^{v}$, at the moment of $t \longrightarrow t+1$. If $x_{\text {last }}^{v}>v_{\max }^{v}$, vehicles at the velocity of $v_{\max }^{v}$ will enter the cellular $\min \left[x_{\text {last }}^{v}-v_{\text {max }}^{v}, v_{\max }^{v}\right]$ with an inflow rate of $a^{v}$. At the point of exiting the road, if $x_{\text {lead }}^{v}>L_{\text {road }}^{v}$, vehicles will leave the road. 


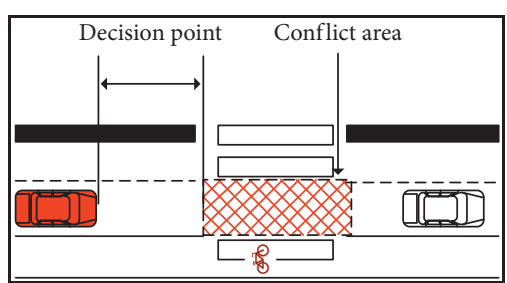

(a)

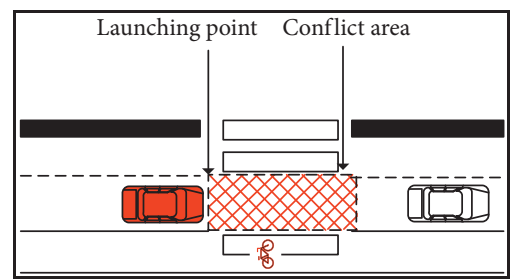

(b)

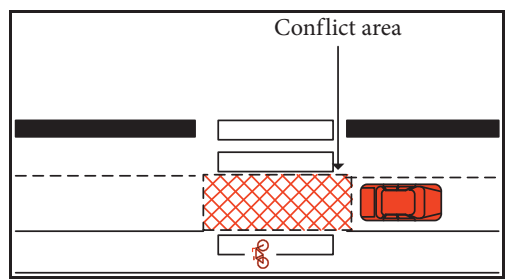

(c)

Figure 2: Schematic diagram of vehicles passing bicycles at crosswalks.

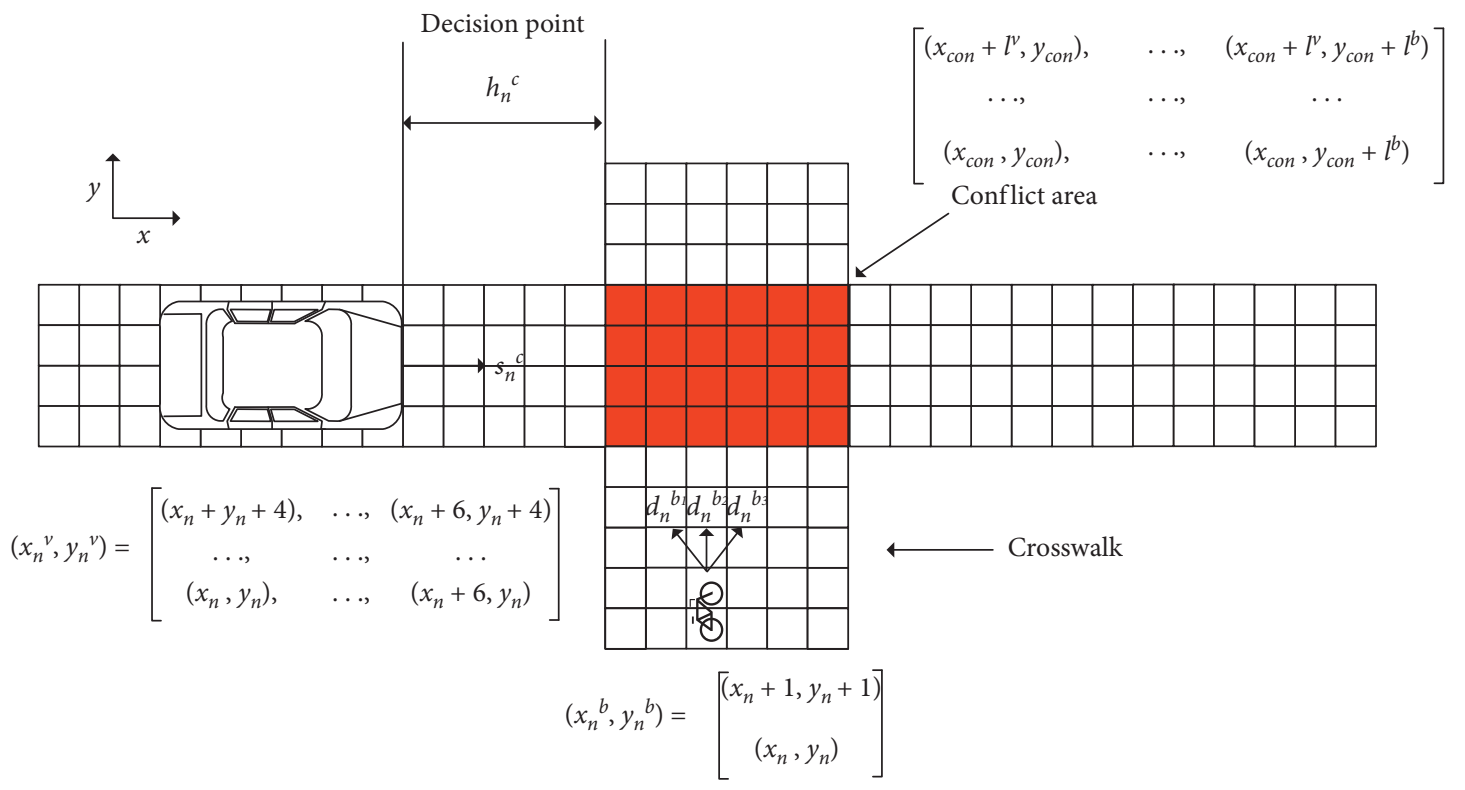

Figure 3: Schematic diagram of mixed-flow cellular space.

3.2.2. Vehicle Rule 2: Decision Point Rule. The decision point is defined as the place where the vehicle driver needs to decide whether or not to give way when encountering a bicycle. In order to mirror the process of a vehicle driver's decision, a deceleration restriction is introduced in the decision point rule and the decision time is a time step. $a^{v}$ is the acceleration and $D$ is the deceleration. It is important to note that the position of the decision point is not a fixed point, but varies with different drivers and is impacted by actual conditions. At the instant of $t$, the decision point is given by

$$
\begin{aligned}
& h_{n}^{v}=v_{n}^{v}+\sum_{i=1}^{t_{n}^{d}}\left(v_{n}^{v}-D \times i\right) \geq x_{v}+l_{b}-x_{n}^{v}, \\
& t_{n}^{d}=\frac{v_{n}^{v}}{D}
\end{aligned}
$$

where $x_{n}^{v}$ and $v_{n}^{v}$ represent the position and velocity of vehicle $n$, respectively; $x_{v}+l_{b}$ is the conflict area boundary; the summation represents the distance from deceleration to stopping; and $t_{n}^{d}$ means the time from deceleration to stop.

When a vehicle is at the decision point, the driver adjusts to a suitable velocity to make sure the cyclist safely traverses the conflict area. Suitable velocity is defined as a velocity that ensures the vehicle not only can pass through the conflict area safely but also can decelerate to a timely stop. In order to capture the stochasticity of different drivers at the decision point, a binary variable $\left(\gamma_{n}^{d}\right)$ is presented as

$$
\gamma_{n}^{d}= \begin{cases}1, & \text { if rand }()>\beta_{1}^{v} \\ 0, & \text { else }\end{cases}
$$

where $\gamma_{n}^{d}=1$ represent the driver choosing to give way to the cyclist; $\gamma_{n}^{d}=0$ represents the driver choosing not to give way, that is, selecting nonstrict priority give way; and $\beta_{1}^{v}$ represents the proportion of nonstrict priority give-way drivers. Thus, the suitable velocity $s_{n}^{v}$ is presented at the instant of $t$ :

$$
s_{n}^{v}= \begin{cases}\min \left(x_{v}-x_{n}^{v}, \max \left(v_{n}^{v}-D, D\right)\right), & \text { Case I, } \\ \left(1-\gamma_{n}^{d}\right) \times \min \left(v_{n}^{v}+a^{v}, v_{\max }^{v}\right)+\gamma_{n}^{d} \times \max \left(v_{n}^{v}-D, D\right), & \text { Case II }\end{cases}
$$


where "Case I" is the vehicle's maximum velocity that will avoid a crash with the bicycle; "Case II" represents the choices of different drivers to give way or not; and $D$ is the deceleration of vehicles.

3.2.3. Vehicle Rule 3: Launching Rule. The vehicle stops at the launching point and waits for an acceptable gap. Once an acceptable gap exists, the driver will take the opportunity to finish the traverse. The acceptable gap is defined as the time interval in which a vehicle successfully traverses the conflict area. In order to capture the stochasticity of different drivers at the launching point, another binary variable $\left(\gamma_{n}^{l}\right)$ is presented as

$$
\gamma_{n}^{l}= \begin{cases}1, & \text { if } \operatorname{rand}()>\beta_{2}^{v}, \\ 0, & \text { else }\end{cases}
$$

where $\gamma_{n}^{d}=1$ represents the driver who needs to give way and waits for bicycles to traverse the conflict area; $\gamma_{n}^{d}=0$ represents the driver who chooses nonstrict priority to give way, for whom the acceptable gap is equal to the launching time of the vehicles; and $\beta_{2}^{v}$ represents the proportion of VNPGWB drivers. Thus, the acceptable gap is given by

$$
t_{n}^{l}=\gamma_{n}^{l}+\left(1-\gamma_{n}^{l}\right) \sqrt{\frac{2 \times l^{b}}{a^{v}}} .
$$

In order to prevent vehicles from remaining static for a long period of time, a threshold vehicle waiting time $T$ is defined. If the waiting time of a vehicle is longer than $T$, the vehicle will launch into the conflict area, and bicycles will be forced to stop.

3.3. Bicycle Model. There is great flexibility in the movement of bicycles, including both lateral and vertical movement. This study chose a new cellular type of bicycle model to simulate one-way bicycle travel in order to explore the interference between bicycles and vehicles in conflict areas. This model consists of two steps, lateral movement and vertical movement, as shown in Figure 3. Both the two steps adopt parallel rules. A bicycle can move to the left, forward, and right: $d_{n}^{l a_{1}}, d_{n}^{l a_{2}}$, and $d_{n}^{l a_{3}}$ describe the number of empty cellular units on the left front, front, and right front, respectively; and $d_{n}^{v e_{1}}, d_{n}^{v e_{2}}$ describe the number of empty cell on the perpendicular left and right, respectively. The bicycle cellular coordinate is represented by $b_{n}=\left(x_{n}^{b}, y_{n}^{b}\right)$, where $b_{n}=0$ means that there is no bicycle occupying this cell; and $b_{n}=1$ means that there is a bicycle occupying this cell; $v_{n}^{b_{l a}}$ represents the lateral velocity of the bicycle; $v_{n}^{b_{v e}}$ represents the vertical velocity of the bicycle, and $v_{n}^{b_{v e}}=-v$ means the bicycle moves to the left; $v_{n}^{b_{\text {ve }}}=v$ means the bicycle moves to the right; $a^{b_{l a}}$ is the lateral acceleration of the bicycle; and $a^{b_{v e}}$ is the vertical acceleration of the bicycle.

3.3.1. Bicycle Rule 1: Lateral Movement. For the process of $t \longrightarrow t+1$, the lateral movement rules are as follows:

Step 1: acceleration; $v_{n}^{b_{l a}} \longrightarrow \min \left(v_{n}^{b_{l a}}+a^{b_{l a}}+v_{\max }^{b_{l a}}\right)$
Step 2: slow down; $d_{n}^{b} \longrightarrow \max \left(d_{n}^{l a_{1}}, d_{n}^{l a_{2}}, d_{n}^{l a_{3}}\right)$, $v_{n}^{b_{l a}} \longrightarrow \min \left(v_{n}^{b_{l a}}+d_{n}^{b}\right)$

Step 3: randomization with probability $p_{s}$; if $\operatorname{rand}()>p_{s}$, then $v_{n}^{b_{l a}} \longrightarrow \max \left(v_{n}^{b_{l a}}+a^{b_{l a}}, 0\right)$

Step 4: movement; $y_{n}^{b} \longrightarrow y_{n}^{b}+v_{n}^{b_{l a}}$

This model adopts an open boundary: when the vehicles' road position is updated, the positions of the head vehicle and the tail vehicle are monitored as $y_{\text {lead }}^{b}$ and $y_{\text {last }}^{b}$, at the moment of $t \longrightarrow t+1$. If $y_{\text {last }}^{b}>v_{\text {max }}^{b}$, bicycles at the speed of $v_{\max }^{b}$ will enter the cellular $\min \left[y_{\text {last }}^{b}-v_{\max }^{b}, v_{\max }^{b}\right]$ with an inflow rate of $a^{b}$. At the exit point of the road, if $y_{\text {lead }}^{b}>L_{\text {road }}^{b}$, the bicycles will exit the road.

3.3.2. Bicycle Rule 2: Vertical Movement. In this study, there are two main situations in which bicycles can move perpendicularly. First, if there is no space in the front, a cyclist can choose vertical movement. Second, if a bicycle moves laterally, vertical movement can be chosen if the number of empty cells on either side is more than or equal to the number in front. The two situations are illustrated in detail as follows:

Situation 1. If $d_{n}^{l a_{1}}=d_{n}^{l a_{2}}=d_{n}^{l a_{3}}=0$, the cyclist chooses vertical movement in order to pass the crosswalk as soon as possible, and $v_{n}^{b_{v e}} \longrightarrow \max \left(d_{n}^{v e_{1}}, d_{n}^{v e_{2}}\right)$. If $d_{n}^{v e_{1}}=d_{n}^{v e_{2}}$, then $\delta_{v e}=\left\{\begin{array}{ll}-1 & \text { if } \operatorname{rand}() \leq p_{v e}^{1} \\ 1 & \text { if } \operatorname{rand}()>p_{v e}^{1}\end{array}\right.$, and $v_{n}^{b_{v e}}=\delta_{v e} \cdot d_{n}^{v e}$.

Situation 2. If $d_{n}^{l a_{1}}, d_{n}^{l a_{2}}$, and $d_{n}^{l a_{3}}$ are not all zero, then the cyclist chooses the largest space in front as far as possible, and $v_{n}^{b_{\text {ve }}}= \begin{cases}-1 & \text { if } d_{n}^{b}=d_{n}^{l_{a_{1}}} \\ 0 & \text { if } d_{n}^{b}=d_{n}^{l_{2}} \text {. If there are two or more } \\ 1 & \text { if } d_{n}^{b}=d_{n}^{l a_{3}}\end{cases}$ choices with the same maximum number of cells, then $\delta_{v e}=\left\{\begin{array}{ll}-1 & \text { if } \operatorname{rand}()<p_{v e}^{2} \\ 0 & \text { if } p_{v e}^{2} \leq \operatorname{rand}() \leq p_{v e}^{3} \\ 1 & \text { if } \operatorname{rand}()>p_{v e}^{3}\end{array}\right.$ and $v_{n}^{b_{v e}}=\delta_{v e} \cdot d_{n}^{v e}$.

3.4. Algorithm for the Mixed-Flow CA Model. In order to simulate the mixed-flow CA model, the abovementioned methods are integrated into one executable algorithm, which is processed in a parallel computing setting using the Python programming environment. The algorithm (Algorithm 1) calculates the process of $t \longrightarrow t+1$. In each step, the following three rules are conducted in order from first to last, and all are applied to every vehicle. When the algorithm ends, the updates are applied to all vehicles and bicycles in parallel. The complete framework of this algorithm's four steps is shown in Figure 4.

\section{Numerical Simulation}

4.1. Simulation Parameter Setting. The data inputs in the following simulations are set as follows. The cellular size is $1 \mathrm{~m} \times 1 \mathrm{~m}$; the vehicle lane length $\left(L^{v}\right)$ is 100 cell units, which therefore corresponds to $100 \mathrm{~m}$; vehicle lane width $\left(l^{v}\right)$ is 4 


\section{Step 1: driving rule.}

Step 1.1: input the current vehicle position $\left(x_{n}^{v}, y_{n}^{v}\right)$; current vehicle velocity $v_{n}^{v}$; a randomization with probability $p_{s}$.

Step 1.2: if the vehicle position is at decision point (equation (1)), then go to Step 2.

Step 1.3: if the vehicle position $x_{n}^{v}+v_{n}^{v}>L_{\text {road }}^{v}$ boundary, then vehicles will leave the road.

Step 1.4: the vehicle adjusts velocity $v_{n}^{v}$ according to the distance $d_{n}^{v}$ to the front vehicle; go to Step 4 .

Step 2: decision point rule.

Step 2.1: input the current bicycle positions $\left(x_{n}^{b}, y_{n}^{b}\right)$ and current bicycle velocities $v_{n}^{b_{l a}}$ and $v_{n}^{b_{v e}}$.

Step 2.2: assume that there exists a time $t_{n}^{1}, 1 \leq t_{n} \leq t_{n}^{d}+1$. If $x_{\text {con }} \leq x_{n}^{b}+v_{n}^{b_{l a}} t_{n}^{1} \leq x_{\text {con }}+l_{v}$ and $y_{\text {con }} \leq y_{n}^{b}+v_{n}^{b_{v e}} t_{n}^{1} \leq y_{\text {con }}+l_{b}$, then the vehicle will crash with bicycles. This vehicle needs to stop at the conflict area boundary and go to Step 3.

Step 2.3: if the driver chooses to give way, then go to Step 2.2; if the driver chooses nonstrict priority give way, then go to Step 4 . The vehicle velocity is given by equation (4).

Step 3: launching rule.

Step 3.1: input the current bicycle positions $\left(x_{n}^{b}, y_{n}^{b}\right)$, current bicycle velocities $v_{n}^{b_{l a}}$ and $v_{n}^{b_{v e}}$, and waiting time $t^{w}$.

Step 3.2: assume that there exists another time $t_{n}^{2}$ and the acceptable gap $t_{n}^{l}$ is given by equation (6). If the driver chooses to give way and $x_{\text {con }} \leq x_{n}^{b}+v_{n}^{b_{l a}}\left(t_{n}^{2}+t_{n}^{l}\right) \leq x_{\text {con }}+l_{v} y_{\text {con }} \leq y_{n}^{b}+v_{n}^{b_{v e}}\left(t_{n}^{2}+t_{n}^{l}\right) \leq y_{\text {con }}+l_{b}$, then the vehicle continues to wait; for waiting time, add 1 ; if the driver chooses nonstrict priority give way, then go to Step 4 . The vehicle velocity $v_{n}^{v}=\left(1-\gamma_{n}^{l}\right) a_{n}^{v}$.

Step 3.3: if the waiting time $t^{w}$ is longer than $\mathrm{T}$, the vehicle will launch into the conflict area, and bicycles will be forced to stop.

Step 4: movement.

Update the position of vehicles $\left(x_{n}^{v}, y_{n}^{v}\right)$ and bicycles $\left(x_{n}^{b}, y_{n}^{b}\right)$ based on their velocities $v_{n}^{v}$ and $v_{n}^{b_{l a}}, v_{n}^{b_{v e}}$. For the vehicle, $x_{n}^{v}(t+1)=x_{n}^{v}(t)+v_{n}^{v}, y_{n}^{v}(t+1)=y_{n}^{v}(t)$. For the bicycle, $x_{n}^{b}(t+1)=x_{n}^{b}(t)+v_{n}^{b_{l a}}, y_{n}^{b}(t+1)=y_{n}^{b}(t)+v_{n}^{b_{v e}}$.

Algorithm 1: The algorithm of mixed-flow CA model.

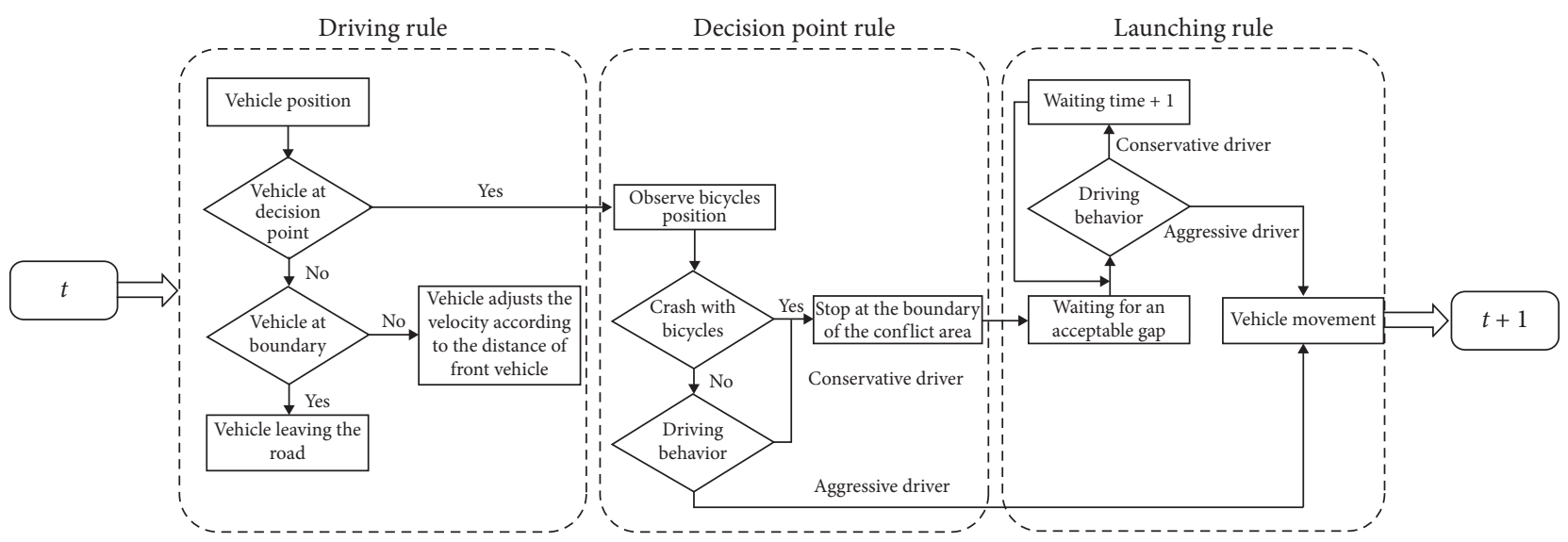

Figure 4: Complete mixed-flow CA model framework.

cells, which corresponds to $4 \mathrm{~m}$; the crosswalk length $\left(L^{b}\right)$ is 100 cell units, and it corresponds to $100 \mathrm{~m}$; crosswalk width $\left(l^{b}\right)$ is 6 cell units, and it corresponds to $6 \mathrm{~m}$ and the size of the conflict area is $6 \times 4$ cell, and it corresponds to $6 \times 4 \mathrm{~m}^{2}$. The simulation parameters are shown in Table 1 . In order to obtain vehicle and bicycle traffic flow data, the virtual detectors are set at the boundaries of the vehicle and crosswalk. When a vehicle or bicycle leaves the lane, the detector's counter adds one. Moreover, $q^{v}$ and $q^{b}$ are the vehicle and bicycle flow, respectively, and the unit is veh/(time $\times$ lane). The simulation steps are $100,000 \mathrm{~s}$, and the preceding $20,000 \mathrm{~s}$ are abandoned.

4.2. Model Validation. To verify the reliability of the mixedflow CA model, the traffic flux-density relationship is the fundamental diagram that describes driver-cyclist interactions. With settings of $\beta_{1}^{v}=0.1, \beta_{2}^{v}=0.1$, and $T=30 \mathrm{~s}$,
TABLE 1: The simulation parameters in mixed-flow CA model.

\begin{tabular}{lcc}
\hline Variable & Vehicle & Bicycle \\
\hline Lane length $(\mathrm{m})$ & $L^{v}=100$ & $L^{b}=50$ \\
Lane width $(\mathrm{m})$ & $l^{v}=4$ & $l^{b}=6$ \\
Max velocity $(\mathrm{m} / \mathrm{s})$ & $v_{\max }^{v}=20$ & $v_{\text {max }}^{b_{l a}}=6$ \\
Acceleration $\left(\mathrm{m} / \mathrm{s}^{2}\right)$ & $a^{c}=4$ & $v_{\text {max }}=2$ \\
Deceleration $\left(\mathrm{m} / \mathrm{s}^{2}\right)$ & $D=10$ & $a^{b_{l a}}=2$ \\
& $p_{s}=0.5$ & - \\
Randomization with probability & & $p_{v e}^{1}=0.5$ \\
& & $p_{v e}^{2}=0.1$ \\
& & $p_{v e}^{3}=0.9$ \\
\hline
\end{tabular}

Figures 5(a) and 6(a) show the microscopic fundamental relationships between flow and inflow rate for the vehicle and bicycle. When the bicycle's inflow rate $\alpha^{b}=0$, there is no bicycle passing the crosswalk, and the vehicle flow $q^{v}$ shows an upward trend and remains stable at 0.34 (Figure 5(b)). When bicycle's inflow rate $\alpha^{b}=1$, the maximal point of 

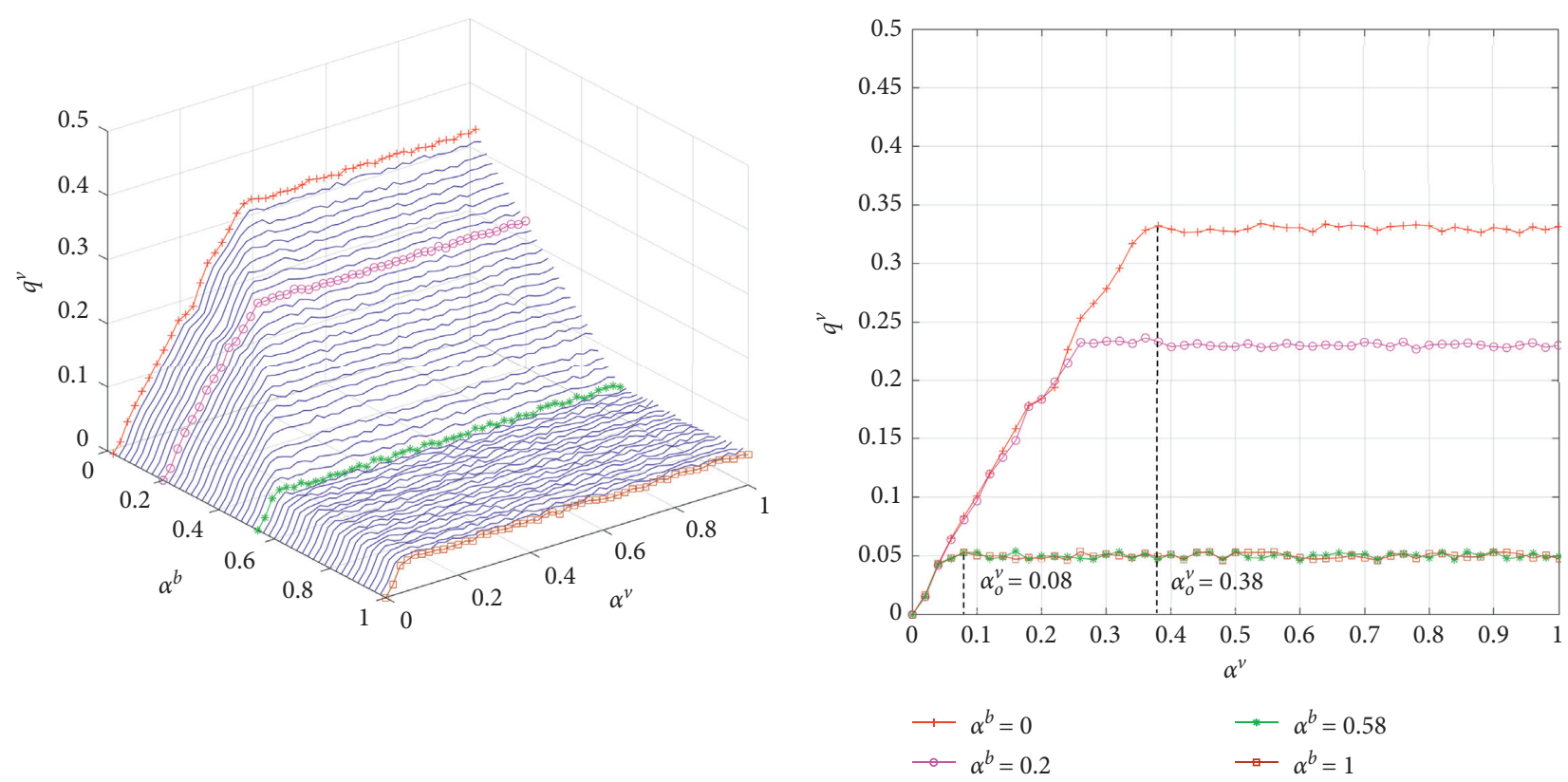

(a)

(b)

Figure 5: The relationship between the vehicle flow and the vehicle and bicycle inflow rate.

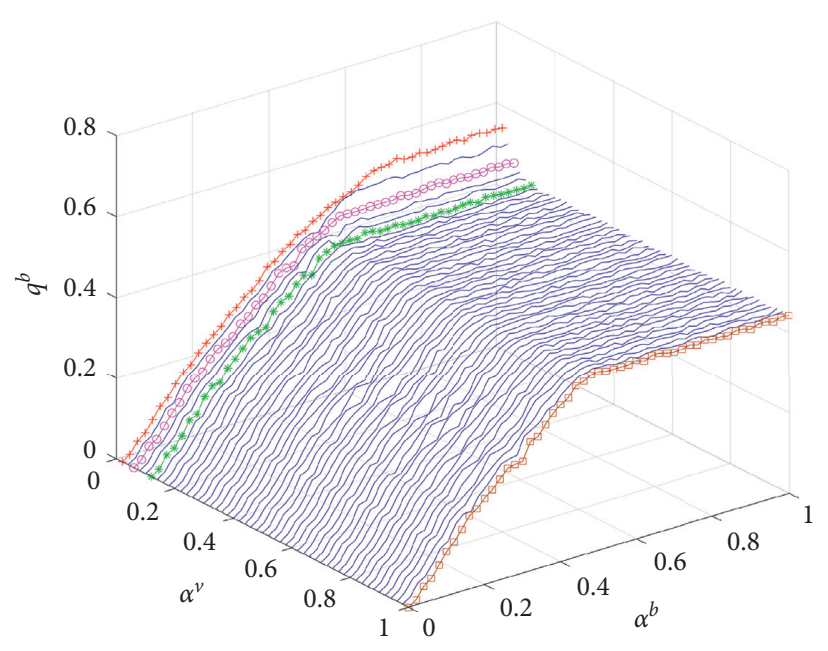

(a)

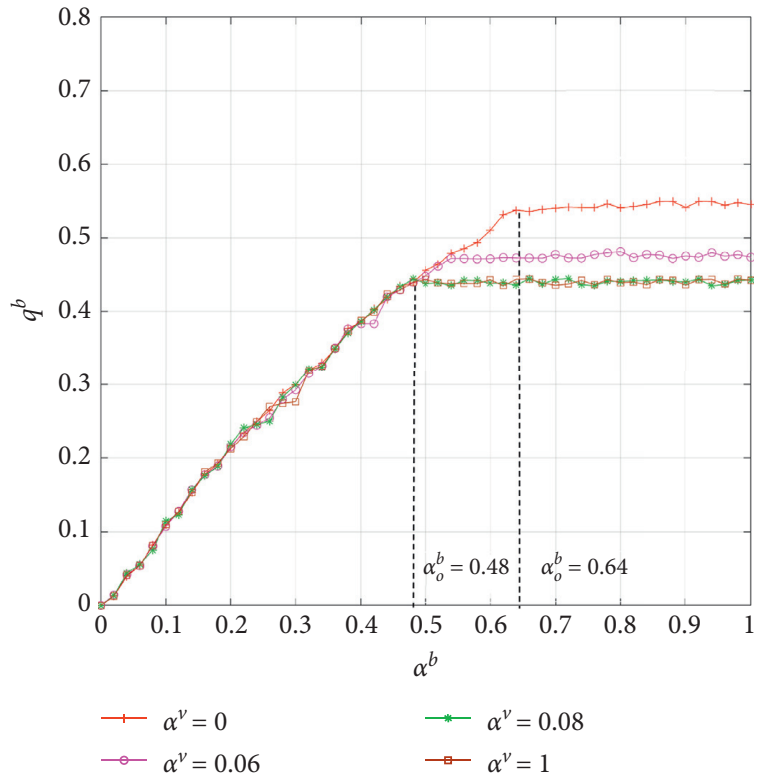

(b)

FIgURe 6: The relationship between the bicycle flow and the vehicle and bicycle inflow rate.

vehicle flow $q_{o}^{v}$ is 0.05 (i.e., saturation flow), which is obviously lower than $\alpha^{b}=0$. With more and more bicycles entering the mixed-flow cellular space, drivers must slow down and stop outside the conflict area to avoid crashes. Thus, only a few vehicles cross the conflict area. However, this situation does not occur for bicycle flow $q^{c}$, because bicycles have priority in passing over the crosswalk. The saturation flow rate of bicycles drops only slightly when bicycles are waiting for vehicles to cross the conflict area. Thus, in the proposed mixed-flow CA model, the transition phase from the free flow to saturation is observed (Figure 7(a)). As shown in Figure 7(a), conflicts between vehicles and bicycles in the traffic system result in a drop in saturated flows, demonstrating that the model can reveal the interactions between vehicles and bicycles in the mixed traffic system.

4.3. Vehicle and Bicycle Flow Transition Phase. As can be seen in Figures 5(a) and 6(a), each curve has an obvious turning point (i.e., critical inflow rate) $\alpha_{o}^{v}\left(\alpha_{o}^{b}\right)$, which divides the flow 


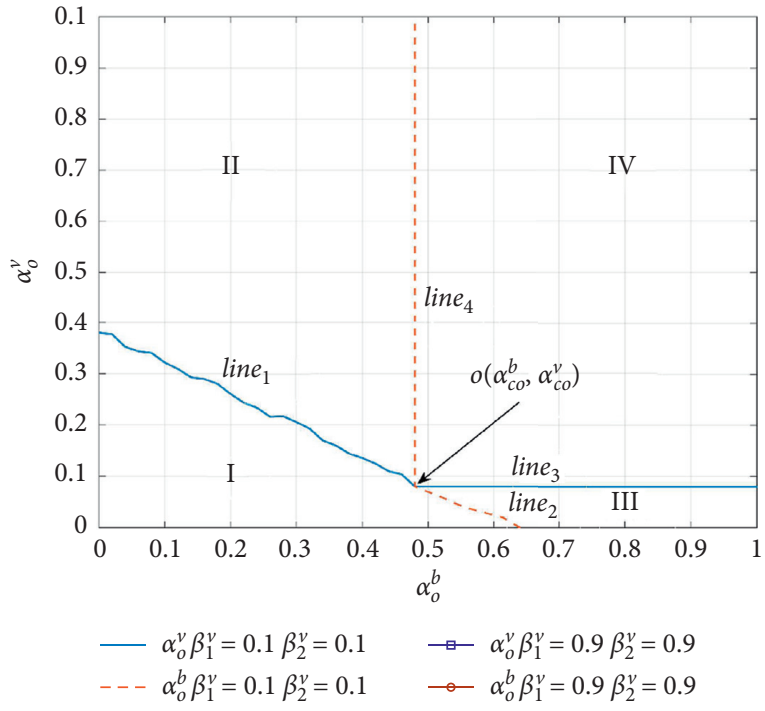

(a)

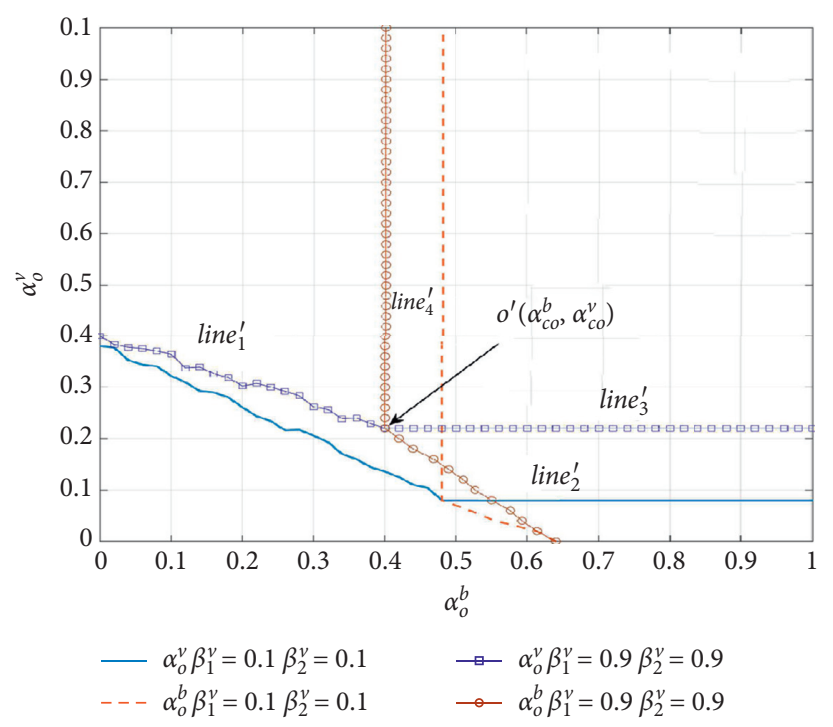

(b)

FIgURE 7: Phase diagram of vehicle and bicycle critical inflow rates.

into two regions: the free flow and saturated flow. This section mainly analyzes the transition phase between the vehicle flow $q_{o}^{v}$ and bicycle flow $q^{c}$.

For $\alpha^{v}<\alpha_{o}^{v}$, the vehicle flow is free flow, and the vehicle flow only depends on its own inflow rate $\alpha^{v}$. Conversely, for $\alpha^{v}>\alpha_{o}^{v}$, the vehicle flow is saturation flow, the vehicle flow $q^{v}$ is independent of its own rate, and it reaches its saturation flow $q_{o}^{v}$, as shown in Figure 5(a). However, with an increase of bicycle inflow rate $\alpha^{b}$, both the critical value of the inflow rate $\alpha_{o}^{v}$ and the saturation flow value $q_{o}^{v}$ diminish until a minimum value is reached. To illustrate the relationship between the inflow rate $\alpha^{v}$ and the vehicle flow $q^{v}$, Figure 5(b) shows four curves of different bicycle inflow rates $\alpha^{b}$. From this diagram, it is evident that the values of vehicle saturation flow $q_{o}^{v}$ decrease from 0.34 to 0.05 , and the critical values of inflow rate $\alpha_{o}^{v}$ decrease from 0.38 to 0.08 . The drop ratio of $q_{o}^{v}$ is about $85 \%$. Similarly, for $\alpha^{b}<\alpha_{o}^{b}$, the bicycle flow is free flow, and for $\alpha^{b}>\alpha_{o}^{b}$, the bicycle flow is saturation flow. The bicycle flow $q^{b}$ is independent of its own rate and reaches its saturation flow value $q_{o}^{b}$, as shown in Figure 6(b). The values of bicycle saturation flow $q_{o}^{b}$ decrease from 0.54 to 0.44 , and the critical values of inflow rate $\alpha_{o}^{b}$ decrease from 0.64 to 0.48 . The drop ratio of $q_{o}^{b}$ is about $19 \%$.

It is noteworthy that the vehicle (bicycle) critical inflow rate $\alpha_{o}^{v}\left(\alpha_{o}^{b}\right)$ gradually decreases with the bicycle (vehicle) inflow rate $\alpha^{b}\left(\alpha^{v}\right)$. Moreover, the collective effect of vehicles and bicycles only appears when $\alpha^{v}$ and $\alpha^{b}$ surpass their critical value. The critical inflow rate $\alpha_{o}^{v}\left(\alpha_{o}^{b}\right)$ is calculated and presented in the phase diagram in Figure 7. This transition phase can be classified into four zones; for example, line $_{1}$ and line $_{3}$ are the boundaries of Zone I (III) and Zone II (IV), which correspond to the critical value of the vehicle inflow rate $\alpha_{o}^{v}$. In Zone I and Zone III, the vehicle is in free flow, whereas in Zone II and Zone IV, it is in saturation flow. As the bicycle inflow rate $\left(\begin{array}{l}b \\ \alpha\end{array}\right)$ increases, the vehicle critical inflow rate $\left(\alpha_{o}^{v}\right)$ first decreases and then remains stable. Similarly, the bicycle critical inflow rate $\alpha_{o}^{b}$ shows the same trend (line 2 and line $_{4}$ ). With the gradual interaction between the two traffic flows, the vehicle's critical inflow rate $\alpha_{o}^{v}$ and bicycle's critical inflow rate $\alpha_{o}^{b}$ reach equilibrium at cross point $o\left(\alpha_{c o}^{b} \alpha_{c o}^{v}\right)$.

In a word, the mixed-flow CA model effectively illustrates the transition phase from free flow to saturation for both vehicles and bicycles. It is interesting that the collective effect of the vehicle flow $q^{v}$ and bicycle saturation flow $q^{b}$ only appears when $q_{o}^{v}$ and $q_{o}^{b}$ surpass their critical inflow rate.

4.4. Comparison between Give-Way and Nonstrict Priority Give-Way Behavior. Demonstrating the effects of VNPGWB in mixed traffic flow, the proportion of VNPGWB drivers $\beta_{1}^{v}$ and $\beta_{2}^{v}$ has increased from 0.1 to 0.9 , respectively. Figure 8 (a) shows the relationships between flow and inflow rate for the vehicle and bicycle in the case of $\beta_{1}^{v}=0.9, \beta_{2}^{v}=0.9$, and $T=30 \mathrm{~s}$. The vehicle saturation flow increases from 0.34 to 0.36 when $\alpha^{b}=0$, while the vehicle saturation flow increases from 0.08 to 0.12 when $\alpha^{b}=1$. When the bicycle inflow rate is low, the VNPGWB driver will quickly pass through the crosswalk, whereas the strict give-way driver will slow down to pass through the crosswalk due to caution. Thus, the vehicle saturation flow resulting from nonstrict priority behavior is slightly higher than from strict give-way behavior. With the further increase of the bicycle traffic flow rate, the VNPGWB driver can pass through the crosswalk more easily than can the strict giveway driver. In this way, VNPGWB can indeed improve the vehicle saturation flow, as the vehicle saturation flow of nonstrict priority is 1.5 times larger than that of strict give way. Figure 8 (b) shows the effect of VNPGWB on vehicle saturation flow in the decision point rule and launching rule. The vehicle saturation flow decreases nonlinearly with an increasing proportion of VNPGWB drivers, with the 


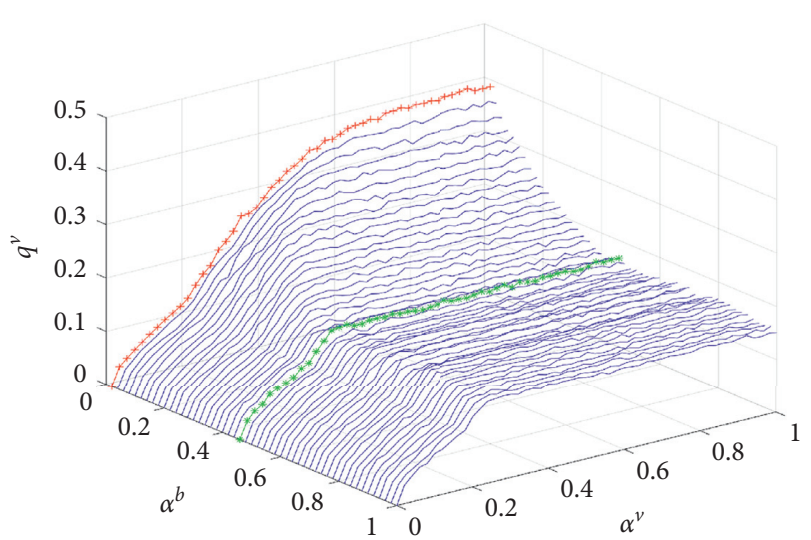

(a)

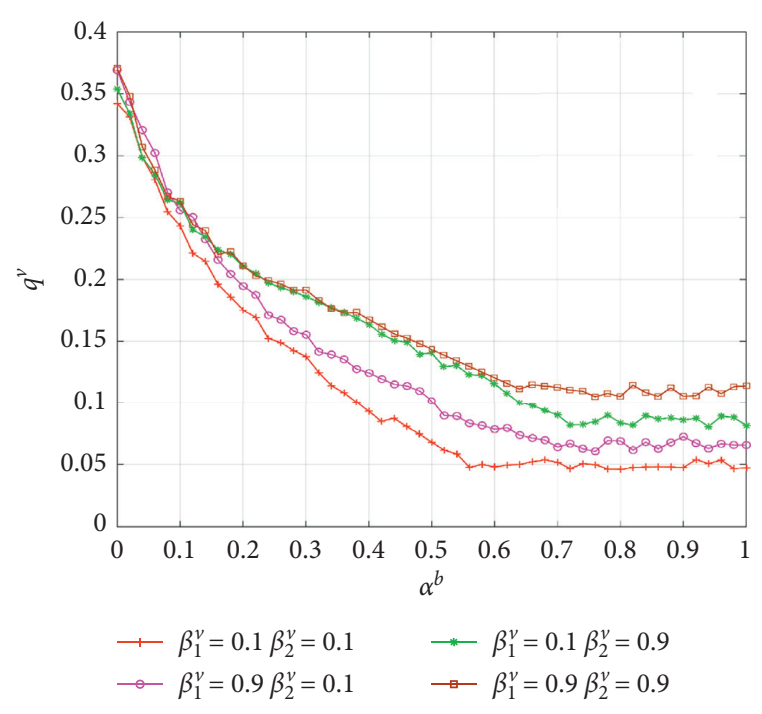

(b)

FIGURE 8: Relationship between vehicle flow and vehicle and bicycle inflow rate under different driving behaviors.

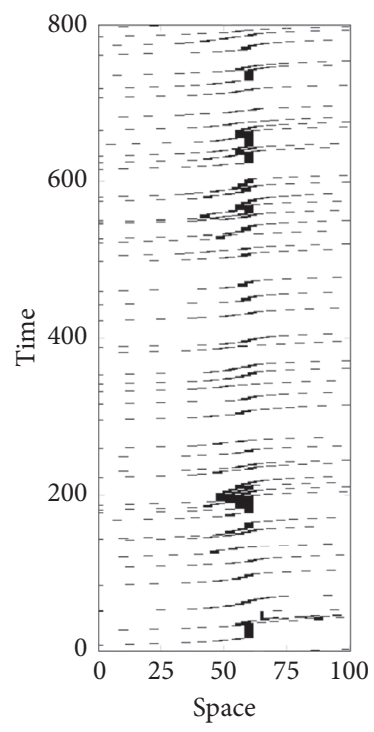

(a)

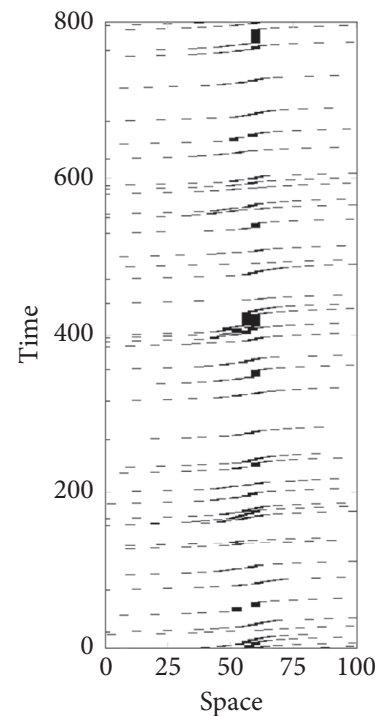

(b)

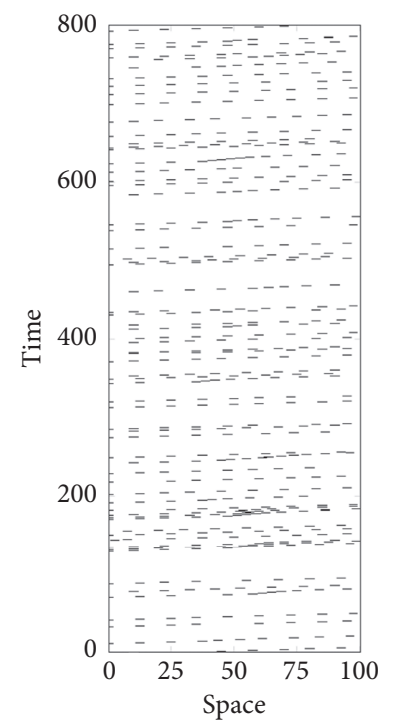

(c)

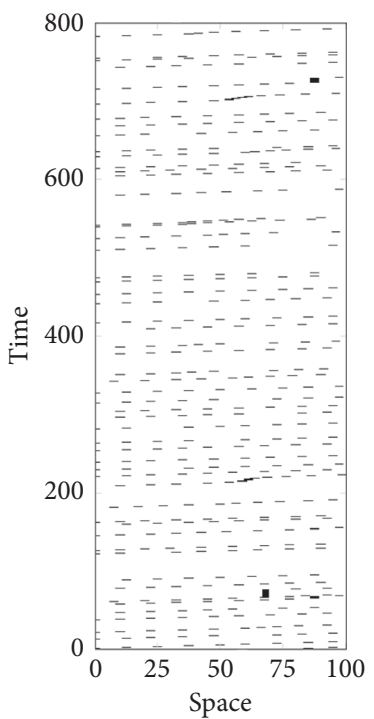

(d)

Figure 9: Space-time diagrams of free flow vehicles under different driving behaviors $\left(\alpha^{v}=0.1, \alpha^{b}=0.1\right)$. (a) $\beta_{1}^{v}=0.1, \beta_{2}^{v}=0.1$. (b) $\beta_{1}^{v}=0.1$, $\beta_{2}^{v}=0.9$. (c) $\beta_{1}^{v}=0.9, \beta_{2}^{v}=0.1$. (d) $\beta_{1}^{v}=0.9, \beta_{2}^{v}=0.9$.

advantage of VNPGWB being more significant in the decision point rule than in launching rule. As shown in Figure 7(b), VNPGWB also affects the vehicle and bicycle critical inflow rates. For $\beta_{1}^{v}=0.9$ and $\beta_{2}^{v}=0.9$, the boundary of line $e_{1}$ and line $e_{3}$ moves up and boundary of line 2 and line 4 moves down. In other words, the proportion of free flow vehicle traffic increases with the increase of VNPGWB.

To further verify that the proposed model can simulate VNPGWB in the real world, the space-time trajectory diagrams for the vehicle lane are shown in Figures 9 and 10. Obviously, the microscopic model proposed in this study is primarily used to describe different microlevel driving behaviors. When the vehicle and bicycle are in free flow
(Figure 9), most vehicles pass through the crosswalk normally, while some drivers choose to slow down and temporarily stop to give way to bicycles at cellular spaces between 40 and 60. As mentioned in Section 3.2.2., the decision point is not a fixed point, which is also depicted in the space-time trajectory diagrams. When the vehicle and bicycle are in free flow, only $\beta_{1}^{v}=0.1$ produces congestion at cellular space 60, and it dissipates very quickly (Figures 9(a) and $9(\mathrm{~b})$ ). On the other hand, when vehicles and bicycles are in saturation flow, most vehicles need to slow down and stop outside the conflict area, as shown in Figure 10. In this case, the congestion cannot dissipate immediately. The $\beta_{2}^{c}$ in the launching rule has a more significant effect on vehicle 


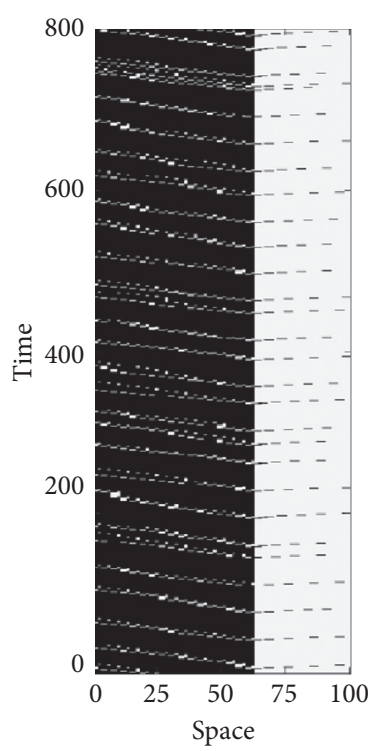

(a)

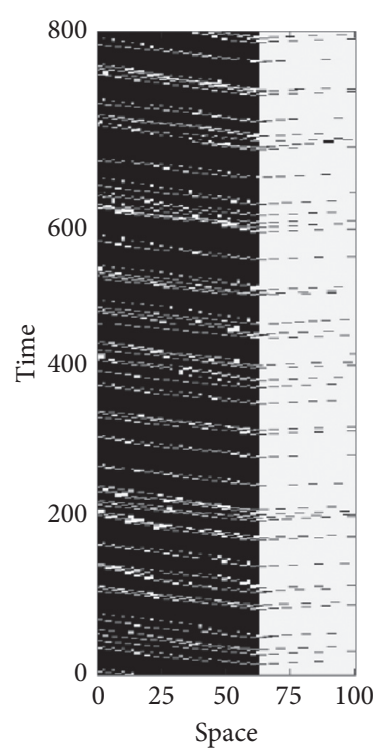

(b)

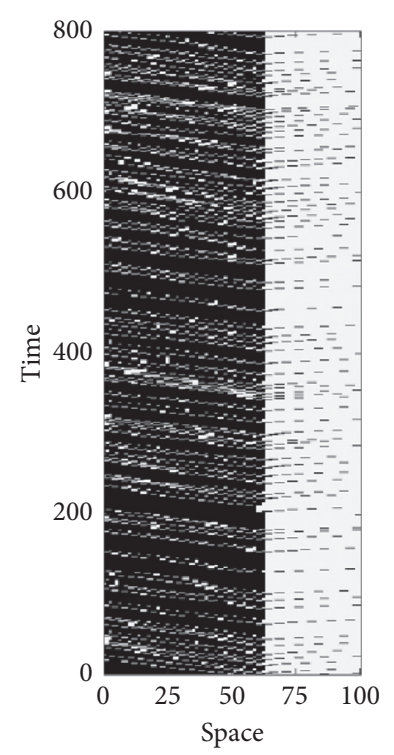

(c)

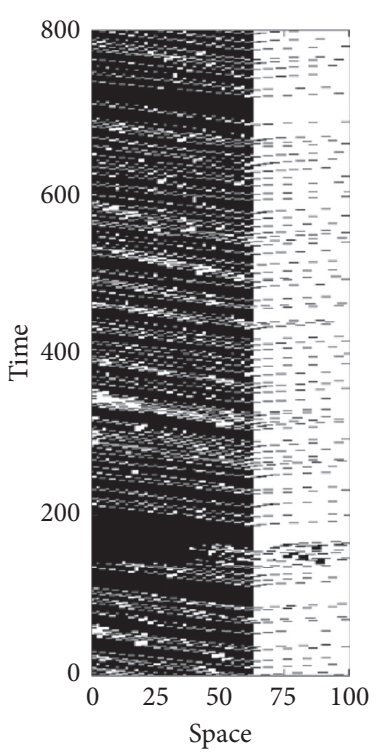

(d)

FIgURe 10: Space-time diagrams of saturation flow vehicles under different driving $\left(\alpha^{v}=0.5, \alpha^{b}=0.5\right)$. (a) $\beta_{1}^{v}=0.1, \beta_{2}^{v}=0.1$. (b) $\beta_{1}^{v}=0.1$, $\beta_{2}^{v}=0.9$. (c) $\beta_{1}^{v}=0.9, \beta_{2}^{v}=0.1$. (d) $\beta_{1}^{v}=0.9, \beta_{2}^{v}=0.9$.

saturation flow than does $\beta_{1}^{c}$ in the decision point rule. Due to bicycle saturation flow, the vehicles form a long queue and waiting for an acceptable gap. For $\beta_{2}^{c}=0.1$, the stopped vehicle can only be launched when its waiting time exceeds the waiting threshold (Figures 10(a) and 10(c)). For $\beta_{1}^{c}=0.9$, some of the space trajectories continue to pass into the conflict area, primarily due to nonstrict priority drivers sometimes choosing to follow the front vehicle through the crosswalk when the bicycle flow is high.

Overall, the space-time trajectory diagrams show that the proposed model in this study effectively simulates the interference between vehicles and bicycles. It is further demonstrated that VNPGWB can improve vehicle flow over strict give way. Especially, when the vehicle and bicycle are in saturation flow, this advantage of VNPGWB is significant. These conclusions can provide support for acquiescence to nonstrict priority behavior for practical management in those countries (e.g., China).

\section{Conclusions}

The crosswalk is one of the most serious conflict areas between vehicles and bicycles. With the aim of improving traffic flow without compromising safety, this study proposed a new mixed-flow cellular automaton model (CA) to simulate the vehicle nonstrict priority give-way behavior (VNPGWB) in the crosswalk conflict area. To consider the driving behaviors in the appropriate proportions, the proposed model simulates VNPGWB in real traffic conditions. This model was divided into three rules: the driving rule, the decision point rule, and the launching rule; VNPGWB occurs in the decision point rule and the launching point rule. The main results of the simulation model are as follows:

(1) The mixed-flow CA model effectively illustrated the transition phase from free flow to saturation flow for both vehicles and bicycles. As the vehicle and bicycle inflow rate increased, there is a critical inflow rate in each curve which divided the traffic flow between free flow and saturated flow. Moreover, the collective effect of vehicles and bicycles appeared only when the inflow rate surpassed its critical value.

(2) Phase diagrams showed that the mixed traffic flow had a negative effect on the saturated flow of both vehicles and bicycles. When the bicycle inflow rate increased from 0 to 1 , vehicle saturation flow values decreased from 0.34 to 0.05 , and the critical values of inflow rate decreased from 0.38 to 0.08 . When vehicle inflow rate increased from 0 to 1 , bicycle saturation flow values decreased from 0.54 to 0.44 , and the critical values of inflow rate decreased from 0.64 to 0.48 .

(3) The simulation results showed that the vehicle saturation flow of nonstrict priority behavior is 1.5 times larger than that of the strict give way, indicating that VNPGWB can improve the vehicle saturation flow. The space-time trajectory diagrams confirmed these results and thereby demonstrate the reliability of the simulation model. The advantage of VNPGWB over strict give way is most significant when the vehicle and bicycle are in saturation flow.

The simulation results, however, still require calibration of observed data to confirm the model's accuracy. Using field data to verify the model's association of parameters is recommended in the future study. In addition, the lack of variation in the size and speed of the simulated vehicles and bicycles in this study was a limitation that will be improved upon in future models. Moreover, the proposed model can also consider machine learning methods such as logistic regression and random forest to predict driving behavior. 
Finally, similar studies can be conducted for the purpose of implementing traffic control at crosswalks with devices such as signal lights or stop signs. Nonetheless, this model provides insights that can be beneficial to traffic engineers and managers.

\section{Data Availability}

The simulation result data used to support the findings of this study are available from the corresponding author upon request.

\section{Conflicts of Interest}

The authors declare that they have no conflicts of interest.

\section{Acknowledgments}

This work was supported by Jiangsu Provincial Key Research and Development Program (Social Development) Project (BE2019713).

\section{References}

[1] C. Yang, W. Wang, X. Shan, J. Jin, J. Lu, and Z. Li, "Effects of personal factors on bicycle commuting in developing countries," Transportation Research Record: Journal of the Transportation Research Board, vol. 2193, no. 1, pp. 96-104, 2010.

[2] S. Cai, X. Long, L. Li, H. Liang, Q. Wang, and X. Ding, "Determinants of intention and behavior of low carbon commuting through bicycle-sharing in China," Journal of Cleaner Production, vol. 212, pp. 602-609, 2019.

[3] C. Wang, C. Xu, J. Xia, and Z. Qian, "The effects of safety knowledge and psychological factors on self-reported risky driving behaviors including group violations for e-bike riders in China," Transportation Research Part F: Traffic Psychology and Behaviour, vol. 56, pp. 344-353, 2018.

[4] L. Yang, Y. Wang, Q. Bai, and S. Han, "Urban form and travel patterns by commuters: comparative case study of whan and Xi'an, China," Journal of Urban Planning and Development, vol. 144, no. 1, 2018.

[5] D. Zhao, G. P. Ong, W. Wang, and X. J. Hu, "Effect of built environment on shared bicycle reallocation: a case study on Nanjing, China," Transportation Research Part A: Policy and Practice, vol. 128, pp. 73-88, 2019.

[6] X. Wu, W. Xiao, C. Deng, D. C. Schwebel, and G. Hu, "Unsafe riding behaviors of shared-bicycle riders in urban China: a retrospective survey," Accident Analysis \& Prevention, vol. 131, pp. 1-7, 2019.

[7] A. P. Silvano, H. N. Koutsopoulos, and X. Ma, "Analysis of vehicle-bicycle interactions at unsignalized crossings: a probabilistic approach and application," Accident Analysis \& Prevention, vol. 97, pp. 38-48, 2016.

[8] Z. Ma, J. Sun, and Y. Wang, "A two-dimensional simulation model for modelling turning vehicles at mixed-flow intersections," Transportation Research Part C: Emerging Technologies, vol. 75, pp. 103-119, 2017.

[9] J. Pucher, R. Buehler, and M. Seinen, "Bicycling renaissance in North America? An update and re-appraisal of cycling trends and policies," Transportation Research Part A: Policy and Practice, vol. 45, no. 6, pp. 451-475, 2011.

[10] L. Bai, P. Liu, Y. Chen, X. Zhang, and W. Wang, "Comparative analysis of the safety effects of electric bikes at signalized intersections," Transportation Research Part D: Transport and Environment, vol. 20, pp. 48-54, 2013.

[11] P. Huertas-Leyva, M. Dozza, and N. Baldanzini, "Investigating cycling kinematics and braking maneuvers in the real world: e-bikes make cyclists move faster, brake harder, and experience new conflicts," Transportation Research Part F: Traffic Psychology and Behaviour, vol. 54, pp. 211-222, 2018.

[12] A. Høye and A. Laureshyn, "SeeMe at the crosswalk: beforeafter study of a pedestrian crosswalk warning system," Transportation Research Part F: Traffic Psychology and Behaviour, vol. 60, pp. 723-733, 2019.

[13] H. C. Manual, HCM2010. Transportation Research Board, National Research Council, Washington, DC, USA, 2010.

[14] N. Chaurand and P. Delhomme, "Cyclists and drivers in road interactions: a comparison of perceived crash risk," Accident Analysis \& Prevention, vol. 50, pp. 1176-1184, 2013.

[15] D. P. Allen, J. E. Hummer, N. M. Rouphail, and J. S. Milazzo, "Effect of bicycles on capacity of signalized intersections," Transportation Research Record: Journal of the Transportation Research Board, vol. 1646, no. 1, pp. 87-95, 1998.

[16] M. Räsänen, I. Koivisto, and H. Summala, "Car driver and bicyclist behavior at bicycle crossings under different priority regulations," Journal of Safety Research, vol. 30, no. 1, pp. 67-77, 1999.

[17] D. Lin, W. Ma, L. Li, and Y. Wang, "A driving force model for non-strict priority crossing behaviors of right-turn drivers," Transportation Research Part B: Methodological, vol. 83, pp. 230-244, 2016.

[18] Q. Bai, Y. Chen, Z. Qu, P. Tao, N. Cao, and Y. Shen, "Nonstrict priority left-turning maneuvers with a permitted phase at signalized: empirical study in China," Journal of Transportation Engineering, Part A: Systems, vol. 143, no. 3, 2017.

[19] Q. Bai, Z. Qu, X. Song, S. Xiong, and N. Cao, "A method for determining the capacity of an exclusive left lane with a permitted phase under nonstrict priority," Advances in Civil Engineering, vol. 2019, Article ID 2357437, 8 pages, 2019.

[20] K. Nagel and M. Schreckenberg, "A cellular automaton model for freeway traffic," Journal De Physique I, vol. 2, no. 12, pp. 2221-2229, 1992.

[21] G. Ren, L. Lu, W. Wang, X. Gong, and Z. Huang, "Microscopic simulation model for pedestrian flow at signalized crosswalks," Transportation Research Record: Journal of the Transportation Research Board, vol. 2434, no. 1, pp. 113-122, 2014.

[22] T.-Q. Tang, Y.-X. Rui, J. Zhang, and H.-Y. Shang, “A cellular automation model accounting for bicycle's group behavior," Physica A: Statistical Mechanics and Its Applications, vol. 492, pp. 1782-1797, 2018.

[23] S. Wolfram, A New Kind of Science, Wolfram Media, Champaign, IL, USA, 2002.

[24] D. Zhao, W. Wang, C. Li, Z. Li, P. Fu, and X. Hu, "Modeling of passing events in mixed bicycle traffic with cellular automata," Transportation Research Record: Journal of the Transportation Research Board, vol. 2387, no. 1, pp. 26-34, 2013.

[25] L. Lu, G. Ren, W. Wang, C.-Y. Chan, and J. Wang, "A cellular automaton simulation model for pedestrian and vehicle interaction behaviors at unsignalized mid-block crosswalks," Accident Analysis \& Prevention, vol. 95, pp. 425-437, 2016.

[26] J.-P. Meng, S.-Q. Dai, L.-Y. Dong, and J.-F. Zhang, "Cellular automaton model for mixed traffic flow with motorcycles," Physica A: Statistical Mechanics and Its Applications, vol. 380, pp. 470-480, 2007. 